\title{
Gravitational torques in spiral galaxies: Gas accretion as a driving mechanism of galactic evolution
}

\author{
D. L. Block ${ }^{1}$, F. Bournaud ${ }^{2,3}$, F. Combes ${ }^{2}$, I. Puerari ${ }^{4}$, and R. Buta ${ }^{5}$ \\ ${ }^{1}$ School of Computational and Applied Mathematics, University Witwatersrand, Private Bag 3, \\ WITS 2050, South Africa \\ 2 Observatoire de Paris, LERMA, 61 Av. de l'Observatoire, 75014 Paris, France \\ 3 École Normale Supérieure, 45 rue d'Ulm, 75005 Paris, France \\ ${ }^{4}$ Instituto Nacional de Astrofísica, Optica y Electrónica, Calle Luis Enrique Erro 1, 72840 Tonantzintla, \\ Puebla, México \\ 5 Dept. of Physics and Astronomy, University of Alabama, Box 870324, Tuscaloosa, Alabama 35487, USA
}

Received 8 July 2002 / Accepted 18 September 2002

\begin{abstract}
The distribution of gravitational torques and bar strengths in the local Universe is derived from a detailed study of 163 galaxies observed in the near-infrared. The results are compared with numerical models for spiral galaxy evolution. It is found that the observed distribution of torques can be accounted for only with external accretion of gas onto spiral disks. Accretion is responsible for bar renewal - after the dissolution of primordial bars - as well as the maintenance of spiral structures. Models of isolated, non-accreting galaxies are ruled out. Moderate accretion rates do not explain the observational results: it is shown that galactic disks should double their mass in less than the Hubble time. The best fit is obtained if spiral galaxies are open systems, still forming today by continuous gas accretion, doubling their mass every 10 billion years.
\end{abstract}

Key words. galaxies: formation - galaxies: evolution - galaxies: spiral - galaxies: fundamental parameters

\section{Introduction}

Bars are a major perturbation of the gravitational potential and a highly efficient engine for the evolution of morphological and chemical properties. In purely stellar disks, bars are robust, long-lived structures (Combes \& Sanders 1981). In reality, however, spiral galaxies contain gas which provokes the dissolution of bars (Bournaud \& Combes 2002, hereafter BC). The bar itself initiates an important radial gas inflow, which destroys barred structure (Pfenniger \& Norman 1990).

Since bars in gaseous disks are dissolved in a few Gyrs, the ubiquity of bars in the local Universe suggests that bars are also renewed. BC painted a scenario wherein continual gas accretion might be a crucial evolutionary factor. This gas settles in the disk, enhancing its self gravity, reducing the influence of the stabilizing central mass concentration, and a second bar develops. In this Letter, we test this hypothesis, by comparing the observed distribution of gravitational torques (in a well-defined galaxy sample) with the distribution predicted by numerical simulations incorporating gas accretion.

Send offprint requests to: D. L. Block, e-mail: block@cam.wits.ac.za

\section{Observational and numerical techniques}

\subsection{Gravitational torques}

The maximal torque over the entire disk is defined by

$Q_{\mathrm{T}}(R)=\frac{F_{\mathrm{T}}^{\max }(R)}{F_{0}(R)}=\frac{\frac{1}{R}\left(\frac{\partial \Phi(R, \theta)}{\partial \theta}\right)_{\max }}{\frac{\mathrm{d} \Phi_{0}(R)}{\mathrm{d} R}}$

where $F_{\mathrm{T}}^{\max }(R)$ represents the maximum amplitude of the tangential force and $F_{0}(R)$ is the mean axisymmetric radial force, inferred from the axisymmetric component of the gravitational potential, $\Phi_{0}$. The potential is inferred from the visible mass only, in both simulations and observations. We then measure $Q_{\mathrm{b}}=\max _{R}\left(Q_{\mathrm{T}}(R)\right)$. $Q_{\mathrm{b}}$ is simply the bar strength in barred galaxies, or the maximal arms torque in unbarred or nearly unbarred galaxies.

\subsection{Observations}

To derive the distribution of $Q_{\mathrm{b}}$, we use near-infrared images from the Ohio State University Bright Galaxy Survey (OSUBGS, Eskridge et al. 2002). The full sample consists of 198 spiral galaxies having total apparent blue magnitude $B_{\mathrm{T}}<12$ and blue isophotal diameter $D_{25}<6^{\prime}$. The technique 
Table 1. Bulge, disk, and halo parameters used in numerical simulations. The halo mass that is accounted for is the mass of dark matter inside the disk radius.

\begin{tabular}{cccc}
\hline \hline Type & $\begin{array}{c}\text { bulge-to-disk } \\
\text { mass ratio }\end{array}$ & $\begin{array}{c}\text { halo-to-disk } \\
\text { mass ratio }\end{array}$ & $\begin{array}{c}\text { abundance } \\
\text { (OSU sample) }\end{array}$ \\
\hline $\mathrm{Sa}$ & 0.9 & 0.3 & $17 \%$ \\
$\mathrm{Sb}$ & 0.4 & 0.5 & $25 \%$ \\
$\mathrm{Sc}$ & 0.2 & 0.7 & $45 \%$ \\
$\mathrm{Sd}$ & 0.1 & 0.9 & $13 \%$ \\
\hline
\end{tabular}

for deriving $Q_{\mathrm{b}}$ from near-IR images is described by Buta \& Block (2001; see also Block et al. 2001), who used the method of Quillen et al. (1994) to transform deprojected, cleaned images into potentials.

Of the 198 OSUBGS galaxies, only 163 are used here for studies of gravitational torques, for reasons detailed in Sect. 4.1. A constant mass-to-light ratio is assumed, i.e, the dark halo is ignored. The disk scale-height and scale-length are assumed to be related by $h_{\mathrm{z}}=h_{\mathrm{r}} / 12$. Also, bulges are assumed to be as flat as the disk; no decompositions to account for the likely rounder shapes of some bulges have been carried out for this study. Such refinements will be considered in a separate study (Buta et al. 2002).

\subsection{Numerical simulations}

We employ a numerical code to study the evolution of bars and spiral arms in galaxies which do, or do not, accrete gas. The simulations include gas dynamics, star formation, and stellar mass-loss. They model a disk, a bulge, and a dark halo. For a complete description, see BC.

We simulate the evolution of four model galaxies, assumed to represent types $\mathrm{Sa}, \mathrm{Sb}, \mathrm{Sc}$ and $\mathrm{Sd}$, with parameters given in Table 1. The proportion of each morphological type in the observed sample is fully accounted for when we analyze the results. We compute the evolution of each model over $15 \mathrm{Gyr}$. We calculate the mean value of $Q_{\mathrm{b}}$ at periods of $100 \mathrm{Myr}$.

\section{Results}

\subsection{Distribution of gravitational torques}

A histogram showing the number of galaxies as a function of their maximal torque is presented in Fig. 1. The most striking feature of the histogram is the depression in the number of galaxies with very low torques $\left(Q_{\mathrm{b}} \sim 0\right)$. That the drop at $Q_{\mathrm{b}} \sim 0$ is not due to selection effects, but is indeed robust, is discussed in Sect. 4.1.

In Fig. 1, one clearly sees that the shape of the observed histogram is not reproduced without gas accretion. Only large accretion rates reproduce the observed histogram (both the drop at $Q_{\mathrm{b}} \sim 0$ and the number of strong bars with $\left.Q_{\mathrm{b}}>0.5\right)$, when the disk mass doubles in 13,10 or 8 Gyrs.

We also compute the deviation between the observed and simulated histograms. If $N_{i}$ is the number of galaxies observed in each torque class $i$, and $N_{i}^{\prime}$ the corresponding value from

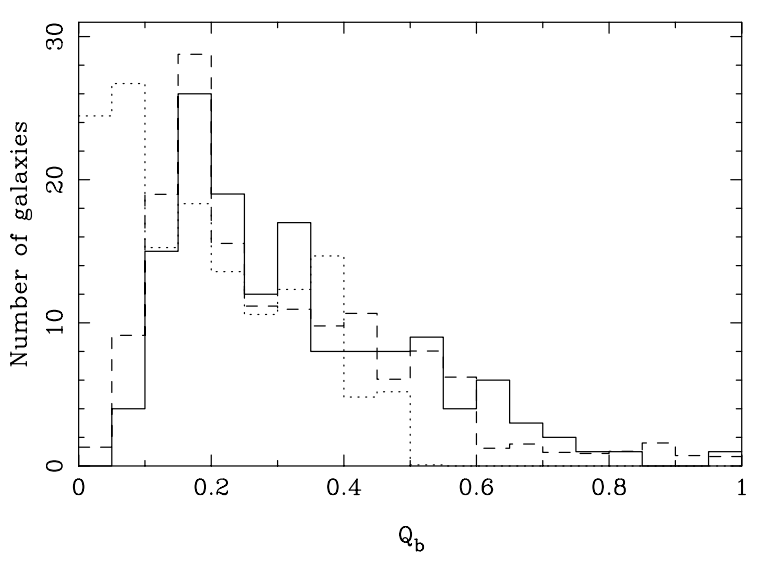

Fig. 1. Histogram of gravitational torques. Solid: observations Dashed: simulations with gas accretion that double the disk mass in 10 Gyrs - Dotted: simulations without gas accretion.

Table 2. Values of $\sigma$ for different accretion rates (in $\mathrm{Gyr}^{-1}$ ), and several gravitational torques and bar strength indicators. $M_{0}$ is the initial disk mass.

\begin{tabular}{cccccc}
\hline \hline Accretion rate & $Q_{\mathrm{b}}$ & $Q_{\mathrm{b}}^{\mathrm{m}}$ & $Q_{2}$ & $Q_{2}^{\mathrm{m}}$ & $F_{\text {bar }}$ \\
\hline no accretion & 1.00 & 1.00 & 1.00 & 1.00 & 1.00 \\
$M_{0} / 30$ & 0.90 & 0.85 & 0.86 & 0.92 & 0.89 \\
$M_{0} / 13$ & 0.37 & 0.45 & 0.51 & 0.39 & 0.32 \\
$M_{0} / 10$ & 0.34 & 0.44 & 0.48 & 0.34 & 0.26 \\
$M_{0} / 8$ & 0.40 & 0.88 & 0.52 & 0.36 & 0.28 \\
\hline
\end{tabular}

simulations, we define $\sigma^{2}=\sum_{i}\left(N_{i}-N_{i}^{\prime}\right)^{2}$. We normalize $\sigma$ to 1 for non accreting models. Values of $\sigma$ are listed in Table 2. The best fit to the observed histogram is obtained when galaxies double their mass every 10 Gyrs, while low accretion rates in which the disk mass doubles in 30 Gyrs, are ruled out.

\subsection{Interpretation: Temporal bar evolution}

The presence of gas in galactic disks is responsible for the destruction of bars in no more than 5 Gyrs. As elucidated by BC, gas is also responsible for bar renewal, when it is accreted from outside the disk. Gas accretion radically changes the temporal evolution of the bar strength: see for example Fig. 4 in BC. With accretion, once a bar is dissolved, the disk can become unstable again and a new bar may form. The disk rarely resides in axisymmetric states: even between bar episodes, spiral arms maintain a significant torque, for accretion also rejuvenates the spiral structure. Without accretion, the disks spend half of their lifetimes in nearly axisymmetric states with $Q_{\mathrm{b}}<0.05$ : both bars and arms disappear. The number of galaxies in each class of the histogram in Fig. 1 is interpreted as the time fraction galaxies spend in each class during their lives.

\section{Discussion}

\subsection{Determination of gravitational torques}

In deriving gravitational torques, we assume that each disk has a constant scale-height. What we measure is thus different from 
the actual torque in nature. However, we compute $Q_{\mathrm{b}}$ exactly in the same manner for the simulations. We adopt the same set of assumptions, which allows a fair comparison between observed and simulated distributions to be made. Even if the value of $Q_{\mathrm{b}}$ is different from the actual maximal torque, the same error will follow through in our simulations.

Obviously, $Q_{\mathrm{b}}$ will differ from the actual torque also because of the dark matter potential. However, the maximal torque is obtained well inside the disk, where the dark matter contribution is negligible. Moreover, $Q_{\mathrm{b}}$ is also computed in simulations ignoring dark matter.

A differentiation between bar and spiral arm torques is not needed. Spiral structure is also present in simulations, so knowing whether $Q_{\mathrm{b}}$ is related to a bar or to a spiral structure is not required.

As mentioned above, only 163 galaxies from 198 in the OSU sample were retained. Our selection criteria are delineated as follows:

- galaxies inclined more than 70 degrees cannot be properly deprojected (deprojection is discussed later);

- members of obvious interacting systems are not studied.

It is unlikely that these criteria are responsible for the depression in the number of galaxies with low torques: in particular, unbarred galaxies were not given preferential rejection.

Thus far, we have itemized several approximations in $Q_{\mathrm{b}}$, but the same assumptions have followed through in the simulations, so that the comparison remains fair. The most questionable point is in fact deprojection, for only observations require such a treatment. An axisymmetric disk could appear non-axisymmetric when the deprojection is not correct: here some approximations may be introduced in observations, but not in simulations. First, observational deprojection assumes that outer disks are circular, which simulations suggest is unlikely. To estimate the resulting uncertainty, we experimented by randomly orienting the simulated images, and deprojecting them exactly as was done for observations. If we denote by $\Delta Q_{\mathrm{b}}$ the change introduced on each bar torque value, we find from simulations that $\sqrt{\left\langle\Delta Q_{\mathrm{b}}{ }^{2}\right\rangle}=.022$, and $\left\langle\Delta Q_{\mathrm{b}}\right\rangle=.007$. A second problem is related to the observational uncertainty of the disk axis ratio $b / a$. We use data from LEDA to determine the uncertainty on $b / a$ as a function of $b / a$. We then convert the result into an uncertainty upon $Q_{\mathrm{b}}$ as a function of the estimated inclination $i$ of the disk, and add to this the first uncertainty on $Q_{\mathrm{b}}$, related to the outer disk shape; values are given in Table 3. Given that the bin of our histogram is 0.05 , the inclination cutoff at 70 degrees is justified, and the results of deprojection are dependable. Another deprojection issue is related to the bar orientation relative to the line of sight: this may affect strongly barred galaxies, but will not affect the crucial number of galaxies with low torques.

\subsection{Other measures of torques and bar strengths}

It is interesting to examine results obtained with physical indicators other than $Q_{\mathrm{b}}$. As mentioned above, $Q_{\mathrm{b}}$ is related either to a bar or to arms, for we select the maximal torque. It may
Table 3. Deprojection uncertainties on gravitational torques $\Delta Q_{\mathrm{b}}$, as a function of the disk inclination $i$.

\begin{tabular}{cccccc}
\hline \hline$i$ & $\Delta Q_{\mathrm{b}}$ & $i$ & $\Delta Q_{\mathrm{b}}$ & $i$ & $\Delta Q_{\mathrm{b}}$ \\
\hline 20 & 0.022 & 50 & 0.025 & 70 & 0.039 \\
35 & 0.023 & 60 & 0.028 & 80 & 0.052 \\
\hline
\end{tabular}

seem better to account for both bar and arms in every disk, by measuring the mean torque over the whole disk:

$Q_{\mathrm{b}}^{\mathrm{m}}=\frac{\int_{r_{\min }}^{r_{\max }} Q_{\mathrm{T}}(r) r \mathrm{~d} r}{\int_{r_{\text {min }}}^{r_{\max }} r \mathrm{~d} r}$

where $r_{\max }$ is the optical radius and $r_{\min }$ is $7 \%$ of $r_{\max }$ (to avoid central singularities). One could also try to eliminate spiral structures and to preferentially focus on bars. To accomplish this, instead of measuring the maximal torque, we measure the torque in the $m=2$ Fourier component: if the potential is decomposed as

$\Phi(r, \theta)=\Phi_{0}(r)+\sum_{m} \Phi_{m}(r) \cos \left(m \theta-\phi_{m}\right)$

we define $Q_{2}(r)=2 \Phi_{2} / r\left|F_{0}(r)\right|$ and $Q_{2}=\max _{r} Q_{2}(r)$. This eliminates spirals with more than 2 arms, thus this parameter may usually be regarded as a measure of bar strength (Combes $\&$ Sanders 1981). Finally, one may incorporate the spatial extent of bars by measuring:

$Q_{2}^{\mathrm{m}}=\frac{\int_{r_{\min }}^{r_{\max }} Q_{2}(r) r \mathrm{~d} r}{\int_{r_{\min }}^{r_{\max }} r \mathrm{~d} r}$.

In contrast, Whyte et al. (2002), have determined the distribution of bar strengths from the OSU sample using bar shapes. They define their bar strength parameter by:

$F_{\text {bar }}=\frac{2}{\pi}\left[\arctan (b / a)^{-\frac{1}{2}}-\arctan (b / a)^{\frac{1}{2}}\right]$

where $b / a$ is the axis ratio of the bar, i.e. the minimum value of the axis ratio of isophotes in an ellipse-fitting model (Abraham et al. 1999). Whyte et al. (2002) also find that nearly axisymmetric disks are rare.

It is difficult to justify one indicator instead of another one; indeed it is important to test our accretion hypothesis with several indicators. The results are given in Table 2. We also show histograms of $F_{\text {bar }}$ in Fig. 2. For other indicators $\left(Q_{2}, Q_{2}^{\mathrm{m}}, Q_{\mathrm{b}}^{\mathrm{m}}\right)$, histograms still show a drop at $Q \sim 0$, which is only reproduced by models with accretion.

All these additional indicators confirm the previous results: the properties of spiral galaxies cannot be reproduced without gas accretion, but require galaxies to double their mass in less than a Hubble time. The best accretion rate makes the mass of spiral disks double over epochs spanning about 10 Gyrs. The difference between the observations and models of closed systems - or galaxies which accrete gas only at a marginal rate - is so flagrant that such models are clearly ruled out. Only large accretion rates reproduce the qualitative shape of the distribution of gravitational torques. Observations of bar axis ratios give the 


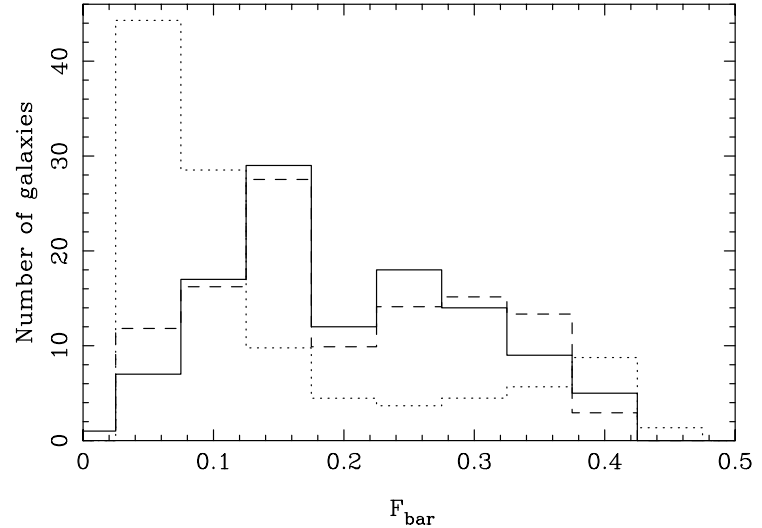

Fig. 2. Histogram of bar strength $F_{\text {bar }}$. Solid: observations, data from Whyte et al. (2002) - Dashed: simulations with gas accretion that doubles the disk mass in 10 Gyrs - Dotted: simulations without gas accretion.

same result. Thus, it seems reasonable to conclude that galaxies accrete large amounts of gas, doubling their mass in less than one Hubble time, even if the exact value we have derived for the accretion rate may need fine-tuning in future studies.

\subsection{Other processes for bar evolution}

We have identified gas accretion as being the process responsible for bar reformation in spiral galaxies. Galaxy interactions may also explain the formation of bars or strengthen existing bars (Gerin et al. 1990). The self-destruction of the first bar heats the disk up and makes it stable. Galaxy interactions would not cool the disk down, a prerequisite for bar reformation and spiral arm maintenance. In the absence of accreted gas, the disk would then become stable because of a large velocity dispersion, thus it would remain unbarred while the spiral structure would become weaker and weaker. Gas accretion, however, keeps the disk cold, and it is then much more susceptible to periodic bar reformation and spiral structure maintenance.

Accretion is thus much more efficient compared to galaxy interactions in maintaining gravitational torques (spirals and bars) in disk galaxies. However, including the influence of galaxy interactions is in preparation. This will allow the distribution of bar strengths from our models (as well as the accretion rates we derive) to be refined, but interactions do not invalidate our present conclusions.

The case of lenticulars will be treated in a separate paper. S0s are stellar systems without much gas. In the absence of gas, the dynamics of disks is different: pure stellar bars are very robust, and can endure for one Hubble time (Combes \& Sanders 1981), contrary to bars in spiral galaxies. In SOs, bars are not destroyed, and no mechanism is needed to explain bar reformation.

\section{Conclusion}

The issue of whether observations support a galaxy dissolving and reforming its bar has awaited the completion of nearinfrared surveys. We are now able to show how robust this scenario is. Isolated galaxies (non-accreting systems) cannot reproduce the observed properties at all: they would become unbarred and spiral arms would disappear; many disks would then be nearly axisymmetric after a few Gyrs. On the contrary, spiral galaxies appear to be open systems that are still forming and continuously accreting mass today. We expect a doubling in disk mass every 10 billion years. The origin of the accreted gas has not been considered, but the most likely source could be the reservoirs of gas observed outside nearly all spiral disks (Sancisi 1983). Pfenniger et al. (1994) have even postulated that the dark matter around spiral galaxies might be in the form of cold gas. Accretion rates from infalling dwarf satellites only represent a few percent of the accretion rate that we derive (Toth \& Ostriker 1992), so that other sources of accretion must be invoked.

Acknowledgements. We acknowledge the valuable comments from an anonymous referee. Funding for the OSU Bright Spiral Galaxy Survey was provided by grants from The National Science Foundation (grants AST-9217716 and AST-9617006), with additional funding by the Ohio State University. We are indebted to P. Eskridge, M. Merrifield and L. Whyte for making the OSU sample available to us in digital format. We have made use of the LEDA database. The research of DLB is funded by the Anglo American Chairman's Fund and SASOL. RB is supported by NSF Grant AST-0205143 to the University of Alabama.

\section{References}

Abraham, R. G., Merrifield, M. R., Ellis, R. S., et al. 1999, MNRAS, 308, 569

Block, D. L., Puerari, I., Knapen, J. H., et al. 2001, A\&A, 375, 761

Bournaud, F., \& Combes, F. 2002, A\&A, 392, 83 (BC)

Buta, R., Laurikainen, E., Salo, H., \& Vasylyev, S. 2002, in preparation

Buta, R., \& Block, D. L. 2001, ApJ, 550, 243

Combes, F., \& Sanders, R. H. 1981, A\&A, 96,164

Eskridge, P. B., et al. 2002, in press [astro-ph/0206320]

Gerin, M., Combes, F., \& Athanassoula, E. 1990, A\&A, 230, 3

Quillen, A. C., Frogel, J., \& Conaález, R. 1994, ApJ, 437, 162

Pfenniger, D., Combes, F., \& Martinet, L. 1994, A\&A, 285, 79

Pfenniger, D., \& Norman, C. 1990, ApJ, 363, 391

Sancisi, R. 1983, Internal Kinematics and Dynamics of Galaxies, IAU Symp., 100, 55

Toth, G., \& Ostriker, J. P. 1992, ApJ, 389, 5

Whyte, L., Abraham, R. G., \& Merrifield, M. R. 2002, MNRAS, in press [astro-ph/0207461] 